Editorial

\title{
Aeroacustic and Vibroacoustic Advancement in Aerospace and Automotive Systems
}

\author{
Roberto Citarella $^{1, *}$ (D) Luigi Federico $^{2}$ and Mattia Barbarino ${ }^{2}$ \\ 1 Department of Industrial Engineering, University of Salerno, 84084 Fisciano, Italy \\ 2 Italian Aerospace Research Centre (C.I.R.A), via Maiorise snc, 81043 Capua, Italy; \\ 1.federico@cira.it (L.F.); m.barbarino@cira.it (M.B.) \\ * Correspondence: rcitarella@unisa.it
}

Received: 6 May 2020; Accepted: 26 May 2020; Published: 1 June 2020

\begin{abstract}
This Special Issue highlights the latest enhancements in the abatement of noise and vibrations of aerospace and automotive systems. The reduction of acoustic emissions and the improvement of cabin interior comfort are on the path of all major transportation industries, having a direct impact on customer satisfaction and, consequently, the commercial success of new products. Topics covered in this Special Issue deal with computational, instrumentation and data analysis of noise and vibrations of fixed wing aircrafts, satellites, spacecrafts, automotives and trains, ranging from aerodynamically generated noise to engine noise, sound absorption, cabin acoustic treatments, duct acoustics and vibroacoustic properties of materials. The focus of this Special Issue is also related to industrial aspects, e.g.,: numerical and experimental studies have been performed for an existing and commercialized engine to enable design improvements aimed at reducing noise and vibrations; moreover, an optimization is provided for the design of low vibroacoustic volute centrifugal compressors and fans whose fluids should be strictly kept in the system without any leakage. Existing procedures and algorithms useful to reach the abovementioned objectives in the most efficient way are illustrated in the collected papers.
\end{abstract}

Keywords: vibroacoustics; aeroacoustics; acoustics; noise; vibration; aeronautics; automotive

\section{Introduction}

This Special Issue follows a previous one [1] on a similar topic, with the aim to keep on providing updates on state of the art research with reference to aeroacoustics and vibroacoustics in aerospace and automotive systems.

In the following, a brief summary on the content of the papers included in this special issue, clustered based on a thematic criterion, starting with the spacecraft vehicles and satellites, then moving to the automotive field, followed by the aeronautic field, the industry components for conditioning and, finally, the railway transport system.

In the section on spacecraft vehicles and satellites, the first paper is concerned with the vibration attenuation of meteorological satellites in the presence of periodic disturbances, leveraging on an optimal design of constant compensations against known-law periodic disturbances [2]. The methodology proposed is applied to a flexible spacecraft actuated by constant control torque in the presence of sustained periodic disturbances. The effectiveness of the optimal compensation torque is provided and compared with results of other selections in the frequency and amplitude ratio domain. Numerical simulation results and experimental results clearly demonstrate the good performance of proposed criteria. In a second paper [3], a frequency weighting matrix beamforming algorithm is proposed to localize air leakages caused by clashes between space debris and spacecraft in orbit. The elastic Lamb waves that are caused by leakages are acquired by an ' $L$ ' shaped sensor array consisting of eight 
acoustic emission sensors. The angle of a leak can be obtained through the superposition of different time delays, and the intersection of two angles can be used to find the location of the leak.

Moving to the automotive field, again a couple of papers can be retrieved. The first analyses the influence of crankshaft torsional frequencies on its rotational speed behavior [4], with the aim to reduce the vibrations at the engine supports. In particular, a multibody calculation methodology has been applied to the vibration analysis of a 4-cylinder, 4-stroke, turbocharged diesel engine, with a simulation-driven study of the angular speed variation of a crankshaft. Time-dependent simulation results, evaluated at the engine supports, are condensed to a vibration index and compared with experimental results, obtaining satisfactory outcomes. The modal analysis also considers the damping aspects and has been conducted using a multibody model created with the software AVL/EXCITE. A second one is concerned with the vibration behavior of a 4-cylinder, 4-stroke, petrol engine as simulated by the finite element method (FEM) [5]. A reduced modelling strategy based on the component mode synthesis (CMS) is adopted to reduce the size of the full FEM model of the engine. Frequency response function (FRF) analyses are used to identify the resonant frequencies and corresponding modes of the different FEM models and the obtained results are compared with experimental data to get the model validation. Subsequently, modal-based frequency forced response analyses have been performed to consider the loads acting during the real operating conditions of the engine. Finally, the impact on vibrations at the mounts, produced by an additional bracket connecting the engine block and gearbox, is investigated. Both the full and reduced FEM model reproduce with high accuracy the vibration response at the engine mounts, but the reduced modelling strategy requires significantly shorter runtimes.

Considering then the aeronautic field, three papers are included in the Special Issue. In one of them, an analytical model to predict the performance of the plasma synthetic jet actuator (PSJA) array, coupled with the multichannel discharge model and PSJA aerodynamic model, is put forward [6]. Such a model can be used to improve the aeroacoustic performances of turbofans by active flow control. In another one [7], the effects of both steady and periodic tangential slot blowing are investigated with the aim to delay buffet onset and alleviate the buffet load. The results show that steady tangential blowing on the airfoil upper surface can postpone the buffet onset margin and evidently increase the lift coefficient at incidence angles near and above the buffet onset case of the clean airfoil. Under buffeting conditions of the clean airfoil, unsteady aerodynamic loads can be greatly suppressed by both steady and periodic blowing. Finally, a novel optimization framework, based on a multi-disciplinary optimization (MDO) procedure, applied to the vibroacoustic finite element method (FEM) model of an aircraft fuselage mock-up, is proposed in [8]. The MDO procedure, based on an efficient global optimization (EGO)-like approach, is implemented to characterize acoustic sources that replicate the sound pressure field generated by the engines on the fuselage. The proposed FEM-MDO framework enables us to set up ground experimental tests on aircraft components, which is useful to replicate their vibro-acoustic performances as if tested in flight. More generally, such a procedure can also be used as a reference tool to design simplified tests starting from more complex ones.

A component adopted for the air conditioning of industrial environments is studied in [9], where a numerical optimization to reduce the vibrational noise of a centrifugal fan volute is presented. Minimal vibrational radiated sound power is considered as the aim of the optimization, and the influence of vibroacoustic coupling is taken into account. The fan's aerodynamic performance, volute casing surface fluctuations, and vibration response have been validated by experiments, showing good agreement. The optimization results show that the vibrational noise optimization method proposed in this study can effectively reduce the vibrational noise of the fan.

The last subsection of this Special Issue is related to railway transport, with particular reference to the aerodynamic noise characteristics of a high-speed train [10]. Considering a pantograph installed on different configurations of the roof base, i.e., flush and sunken surfaces, the large eddy simulation (LES) is coupled with the acoustic finite element method (FEM) to analyze its noise impact. Numerical results are presented in terms of acoustic pressure spectra and distributions of aerodynamic noise in near-field 
and far-field regions. The results show that the pantograph with the sunken base configuration provides better aerodynamic noise performances when compared to that with the flush base configuration.

Author Contributions: R.C., L.F. and M.B. contributed with the same level of involvement in managing the review process for the papers considered for publication in this special issue. All authors have read and agreed to the published version of the manuscript.

Funding: This research received no external funding.

Acknowledgments: The editors would like to express their thanks to all authors of the Special Issue for their valuable contributions and to all reviewers for their useful efforts to provide valuable reviews. We expect that this Special Issue offers a timely view of advanced topics in aeroacoustics and vibroacoustics in aerospace and automotive systems, which will stimulate further novel academic research and innovative applications.

Conflicts of Interest: The authors declare no conflict of interest.

\section{References}

1. Citarella, R.; Federico, L. Advances in Vibroacoustics and Aeroacustics of Aerospace and Automotive Systems. Appl. Sci. 2018, 8, 366. [CrossRef]

2. Xu, S.; Cui, N.; Fan, Y.; Guan, Y. A Study on Optimal Compensation Design for Meteorological Satellites in the Presence of Periodic Disturbance. Appl. Sci. 2018, 8, 1190. [CrossRef]

3. Qi, L.; Zeng, Z.; Zhang, Y.; Sun, L.; Rui, X.; Li, X.; Wang, L.; Liu, T.; Yue, G. Research on Leakage Location of Spacecraft in Orbit Based on Frequency Weighting Matrix Beamforming Algorithm by Lamb Waves. Appl. Sci. 2020, 10, 1201. [CrossRef]

4. Armentani, E.; Caputo, F.; Esposito, L.; Giannella, V.; Citarella, R. Multibody Simulation for the Vibration Analysis of a Turbocharged Diesel Engine. Appl. Sci. 2018, 8, 1192. [CrossRef]

5. Armentani, E.; Giannella, V.; Citarella, R.; Parente, A.; Pirelli, M. Substructuring of a Petrol Engine: Dynamic Characterization and Experimental Validation. Appl. Sci. 2019, 9, 4969. [CrossRef]

6. Huang, S.; Zhang, Z.; Song, H.; Wu, Y.; Sun, Z.; Li, Y. Analytic Model and the Influence of Actuator Number on the Performance of Plasma Synthetic Jet Actuator Array. Appl. Sci. 2018, 8, 1534. [CrossRef]

7. Dang, H.; Zhao, J.; Yang, Z.; Dang, H. Postponing the Onset and Alleviating the Load of Transonic Buffet by Using Steady and Periodic Tangential Slot Blowing. Appl. Sci. 2019, 9, 4132. [CrossRef]

8. Giannella, V.; Lombardi, R.; Pisani, M.; Federico, L.; Barbarino, M.; Citarella, R. A Novel Optimization Framework to Replicate the Vibro-Acoustics Response of an Aircraft Fuselage. Appl. Sci. 2020, 10, 2473. [CrossRef]

9. Chu, W.; Zhang, J.; Lv, Y. Vibroacoustic Optimization Study for the Volute Casing of a Centrifugal Fan. Appl. Sci. 2019, 9, 859. [CrossRef]

10. Yao, Y.; Sun, Z.; Yang, G.; Liu, W.; Prapamonthon, P. Analysis of Aerodynamic Noise Characteristics of High-Speed Train Pantograph with Different Installation Bases. Appl. Sci. 2019, 9, 2332. [CrossRef] 